\title{
A novel immunopathological association of IgG4-RD and vasculitis with Hashimoto's thyroiditis
}

\author{
Hiroto Minamino ${ }^{1,2}$, Hidefumi Inaba', Hiroyuki Ariyasu', Hiroto Furuta', Masahiro Nishi', \\ Takashi Yoshimasu $^{3}$, Akinori Nishikawa ${ }^{4}$, Masanori Nakanishi ${ }^{5}$, Shigeki Tsuchihashi ${ }^{6}$, \\ Fumiyoshi Kojima7, Shin-ichi Murata ${ }^{7}$, Gen Inoue ${ }^{2}$ and Takashi Akamizu ${ }^{1}$ \\ ${ }^{1}$ The First Department of Medicine, Wakayama Medical University, 811-1, Kimiidera, Wakayama, $641-8509$ Japan \\ ${ }^{2}$ Department of Diabetes and Endocrinology, Wakayama Red Cross Hospital, Wakayama, Japan \\ Departments of ${ }^{3}$ Dermatology, ${ }^{4}$ Hematology, ${ }^{5}$ Respiratory Medicine \& Medical Oncology, ${ }^{6}$ Otolaryngology, \\ ${ }^{7}$ Human Pathology, Wakayama Medical University, Wakayama, Japan
}

\author{
Correspondence \\ should be addressed \\ to $\mathrm{H}$ Inaba \\ Email \\ inaba@wakayama-med.ac.jp
}

\section{Summary}

A 73-year-old man with Hashimoto's thyroiditis (HT) suffered from purpura on the lower legs. He was diagnosed with IgG4-related disease (IgG4-RD) with serum IgG4 elevation and dacryo-sialadenitis confirmed histologically. Serum Th2 and Treg cytokines, interleukin 7 (IL7), IL8 and Th2 chemokine levels were elevated, while skewed Th1 balance was seen in fluorescence-activated cell sorting (FACS). Therefore, preferential Th1 balance in HT appeared to be followed by IgG4-RD characterized with Th2 and Treg polarization. The commencement of steroid therapy dramatically exacerbated clinical manifestations including IgG4-RD-associated HT. The measurement of cytokine and chemokine levels as well as FACS analysis in the development of IgG4-RD seemed to be beneficial. In conclusion, an innovative association of HT, IgG4-RD and vasculitis was observed. This report also offers novel diagnostic and therapeutic approaches for IgG4-RD.

\section{Learning points:}

- Recently, a subtype of HT has been considered to be a thyroid manifestation of IgG4-RD, although the etiology of IgG4-RD is not established yet.

- Immunologically a close association between HT and vasculitis was reported.

- Leukocytoclastic vasculitis is a rare skin presentation of IgG4-RD.

- In the current case, during the course of HT, IgG4-RD and leukocytoclastic vasculitis occurred; thus, innate immunity and acquired immunity seem to be involved in the development of IgG4-RD.

- The measurement of cytokine and chemokines appeared to be beneficial in the development of IgG4-RD.

- Remarkably, effectiveness of steroid therapy for HT suggested presence of IgG4-RD-associated HT. Therefore, this report highlights the pathogenesis of IgG4-RD and proposes novel therapeutic mechanisms. Clinicians should pay attention to the development of IgG4-RD and vasculitis during long course of HT.

\section{Background}

IgG4-related disease (IgG4-RD) is a recently proposed clinical entity, characterized by elevated serum IgG4 levels and IgG4-bearing plasmacytes, yet little is known about skin manifestations of IgG4-RD (1). In relation to IgG4-RD and Hashimoto's thyroiditis (HT), a subtype of HT was suggested to be associated with IgG4-RD (2). However, steroid therapy for IgG4-RD-associated HT has been 
controversial. The current case exhibited rare presentation of IgG4-RD with its skin manifestation as leukocytoclastic vasculitis, precisely observed in the long course of HT. In addition to the clinicopathological examination, serum cytokines (Th1, Th2 and Treg); interleukin 7 (IL7), IL8 and Th2 chemokine; and monocyte chemotactic protein 1 (CCL2, also referred to as MCP1) levels were evaluated. This report describes a new association of IgG4-RD and vasculitis concomitant with $\mathrm{HT}$, and highlights effective diagnostic approaches and treatment outcome in IgG4RD-associated HT.

\section{Case presentation}

A 73-year-old man has been treated for $\mathrm{HT}$ with $\mathrm{L}^{-\mathrm{T}_{4}}$ supplementation: $125 \mu \mathrm{g} /$ day for 20 years. Before 6 months, he was diagnosed with interstitial pneumonia. He presented with purpura on both lower legs (Fig. 1) for 1 month and visited our hospital. On admission, his areas of eyelid and salivary glands were symmetrically enlarged. He had a smoking history, $40 \times 30$ years and was a chance drinker. His past history was unremarkable. His body height was $168 \mathrm{~cm}$, and his weight was $66 \mathrm{~kg}$. His blood

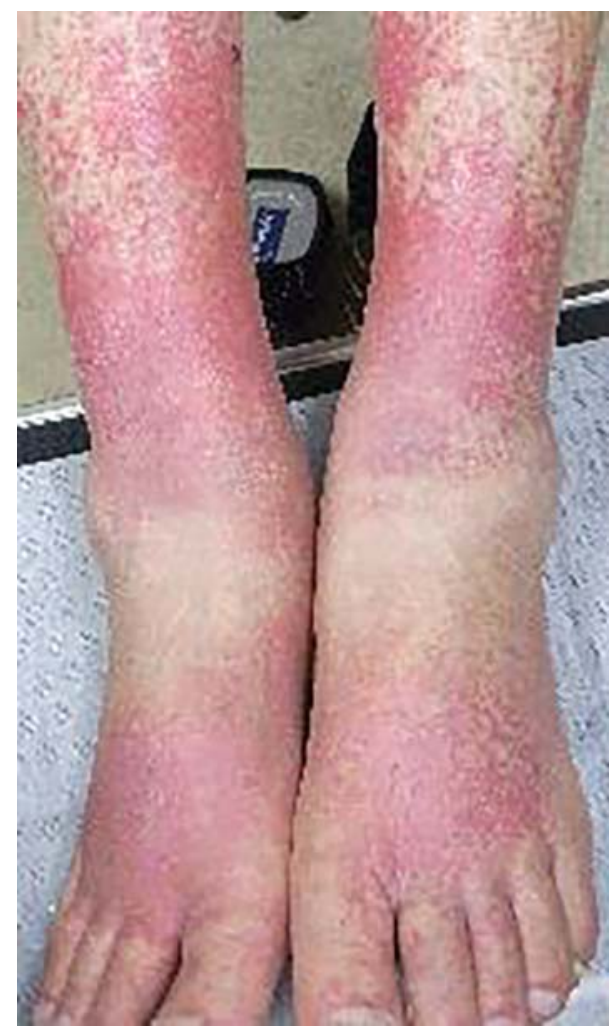

Figure 1

The purpura on both lower extremities was observed. pressure was $124 / 67 \mathrm{mmHg}$, his heart rate was 75 beats/min and regular and his body temperature was $36.5^{\circ} \mathrm{C}$. His thyroid gland was firm, not enlarged. No abnormal heart or lung sounds were detected.

Table 1 Laboratory data and results from endocrinological and immunological tests on admission. Bold values denote abnormal values.

\begin{tabular}{l}
\hline Parameters \\
\hline Laboratory data \\
WBC \\
Hb \\
Plt \\
AST \\
ALT \\
$\gamma$-GTP \\
ALP \\
BUN \\
Cr \\
T-bil \\
CRP \\
D-dimer \\
IgG \\
IgG4 \\
ANA \\
C3 \\
C4 \\
CH 50 \\
C1q \\
Anti-skin antibody \\
PR3-ANCA \\
MPO-ANCA \\
KL-6 \\
SP-A \\
SP-D \\
sIL2R
\end{tabular}

\begin{tabular}{c} 
Values \\
$10620 \mu \mathrm{l}$ \\
$11.6 \mathrm{~g} / \mathrm{dl}$ \\
$27.5 \times 10^{4} \mu \mathrm{l}$ \\
$56 \mathrm{IU} / \mathrm{l}$ \\
$30 \mathrm{IU} / \mathrm{l}$ \\
$41 \mathrm{IU} / \mathrm{l}$ \\
$285 \mathrm{IU} / \mathrm{l}$ \\
$20 \mathrm{mg} / \mathrm{dl}$ \\
$1.06 \mathrm{mg} / \mathrm{dl}$ \\
$0.8 \mathrm{mg} / \mathrm{dl}$ \\
$1.38 \mathrm{mg} / \mathrm{dl}(<0.30)$ \\
$0.50 \mu \mathrm{g} / \mathrm{ml}(<0.30)$ \\
$5554 \mathrm{mg} / \mathrm{dl}(870-1700)$ \\
$897 \mathrm{mg} / \mathrm{dl}(4-108)$ \\
$2560, \mathrm{nucleolar}, \mathrm{cytoplasmic}$ \\
$22 \mathrm{mg} / \mathrm{dl}(65-135)$ \\
$1 \mathrm{mg} / \mathrm{dl}(13-35)$ \\
$<15 \mathrm{U} / \mathrm{ml}(30-50)$ \\
$98 \mu \mathrm{g} / \mathrm{ml}(0-3)$ \\
$(-)$ \\
$1.5 \mathrm{U} / \mathrm{ml}(<2)$ \\
$<0.5 \mathrm{U} / \mathrm{ml}(<3.5)$ \\
$328 \mathrm{U} / \mathrm{ml}(<500)$ \\
$62.8 \mathrm{ng} / \mathrm{ml}(<43.8)$ \\
$118.8 \mathrm{ng} / \mathrm{ml}(<110)$ \\
$2282 \mathrm{U} / \mathrm{ml}(145-519)$ \\
\hline
\end{tabular}

Endocrinological and immunological tests

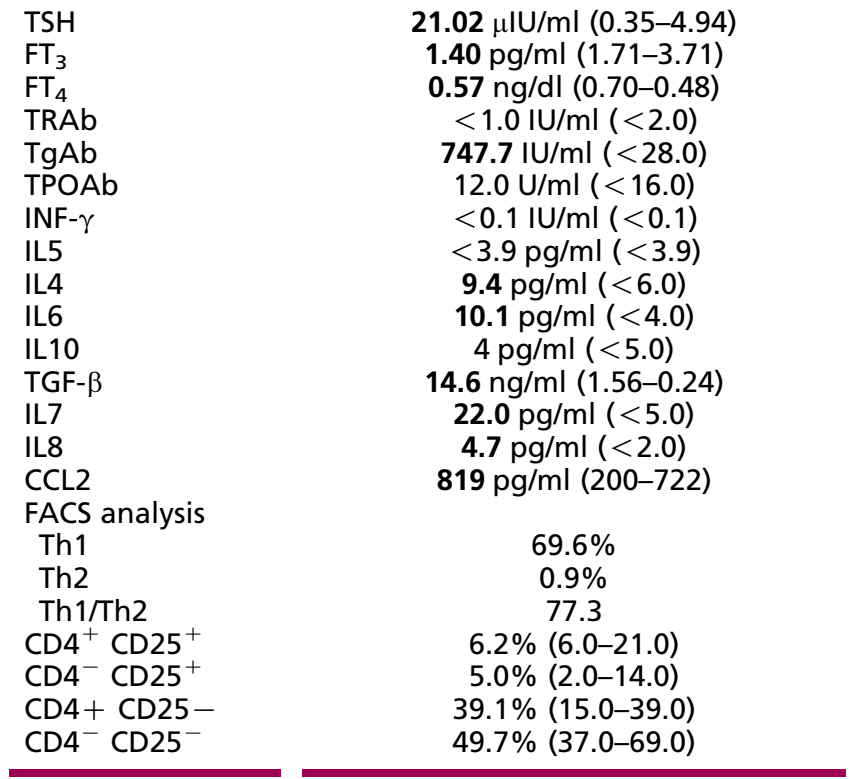

TRAb, TSH receptor antibody (third generation). 


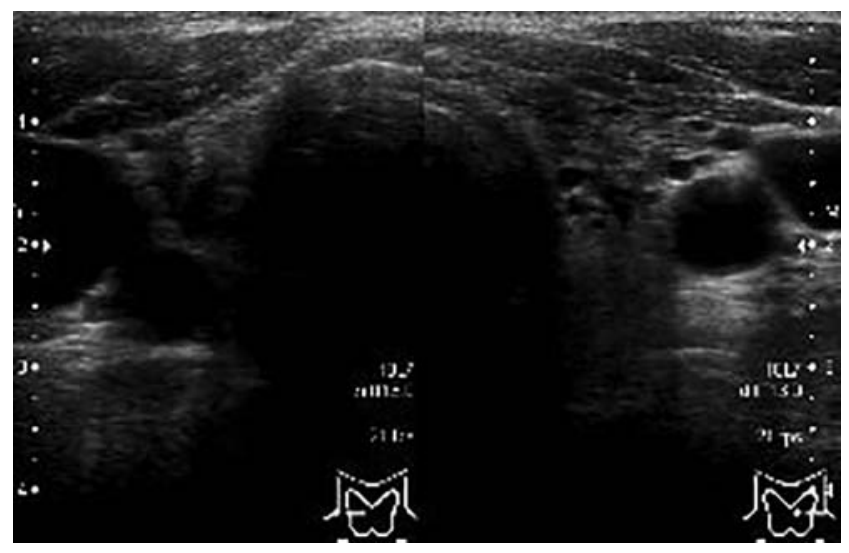

Figure 2

An ultrasonographic examination of the thyroid gland showed dishomogeneous echoic pattern.

\section{Investigation}

In the laboratory test (Table 1 ), serum $\mathrm{C}$-reactive protein (CRP) level was mildly elevated. The serum IgG and IgG4 levels were remarkably elevated. Although antinuclear antibodies were elevated, individual antibodies suggesting collagen diseases were all negative. Hypocomplementemia with elevated $\mathrm{C} 1 \mathrm{q}$ levels was observed. Anti-skin antibodies, PR3-ANCA and MPO-ANCA, were negative. The soluble IL2 receptor (sIL2R) level was elevated. The levels of SP-A and SP-D were also elevated. Cryoglobulins were negative. In endocrinological examinations (Table 1), thyrotropin (TSH) levels were increased; triiodothyronine $\left(\mathrm{FT}_{3}\right)$ and thyroxine $\left(\mathrm{FT}_{4}\right)$ levels were also decreased under a medication of $\mathrm{L}^{-\mathrm{T}_{4}}$ : $50 \mu \mathrm{g} /$ day. Anti-thyroglobulin antibody (TgAb) levels were elevated. In the serum cytokine and chemokine measurement, Th2
(IL4 and IL6), Treg cytokine (transforming growth factor $\beta$ (TGF- $\beta$ )), IL7, IL8 and Th2 chemokine (CCL2) levels were elevated, whereas Th1 cytokine levels (interferon (IFN)- $\gamma$ ), were not increased. In the fluorescence-activated cell sorting (FACS) analysis of peripheral blood mononuclear cell (PBMC), the ratio of Th1/Th2 was increased, and the proportion of $\mathrm{CD} 4{ }^{+} \mathrm{CD} 25^{+}$lymphocytes (suggesting as Treg fraction) was within normal range.

On an ultrasonographic examination, his thyroid gland showed heterogeneous, hypoechogenic pattern (Fig. 2). Orbital CT showed markedly swollen bilateral lacrimal glands (Fig. 3A). On cervical CT, parotid and submandibular glands were symmetrically enlarged (Fig. 3B and $\mathrm{C}$ respectively). Although no abnormalities were seen in the X-ray taken 3 years before (Fig. 4A), mediastinal lymphadenopathy and thicken subpleural interstitial region with peribronchovascular consolidation were observed in chest CT and X-ray on admission (Figs 3D and 4B). Pancreas was not enlarged. FDG accumulation on PET was observed in the lacrimal glands, parotid glands, submandibular glands and pulmonary consolidation (figures not shown). FDG accumulation in the thyroid was not observed. The biopsy specimen of labial gland exhibited diffuse lymphoplasmacytic infiltration containing IgG4 positive plasma cells (the ratio of IgG4/IgG was $96 / 161=59.6 \%$ ), with slight fibrosis, fulfilled the criteria of IgG4-RD (Fig. 5A, B, C). Skin biopsy of the purpura revealed leukocytoclastic vasculitis (Fig. 6A). IgG or IgG4 deposits around the vessel wall were not clear (Fig. 6B and $\mathrm{C}$ ). Although hypocomplementemia was seen, no manifestation suggesting autoimmune diseases and cryoglobulinemia were observed. Allergic purpura was less likely, because of no abdominal pain or arthralgia was seen. Drug-induced purpura was less likely, because no suspected drug was seen.
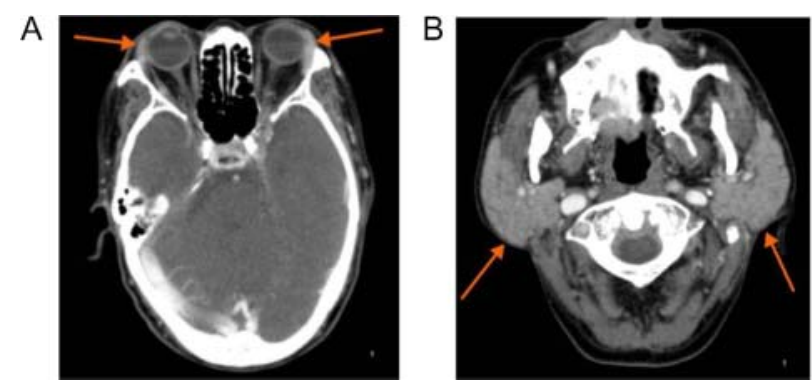

Figure 3

Orbital CT showed (A) in axial image, bilateral lacrimal glands were swelling (arrows) and bilateral proptoses were seen. On cervical CT, parotid glands were symmetrically enlarged (arrows, B). In addition, both
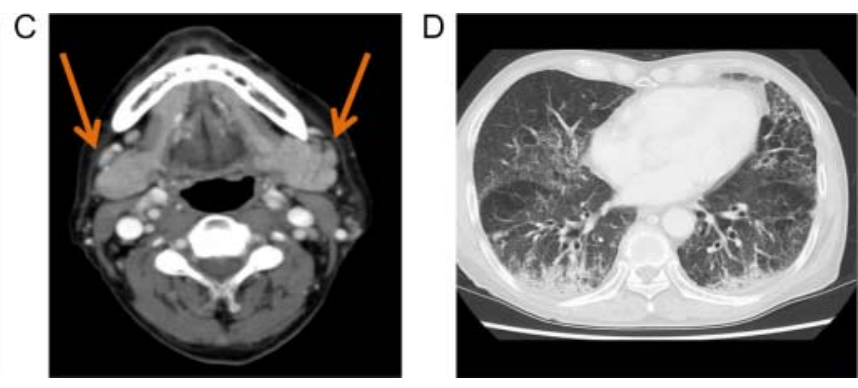

submandibular glands were also enlarged (arrows, C). In chest CT, mediastinal lymphadenopathy with dorsal subpleural peribronchovascular consolidation and thicken bronks were seen in lung (D). 


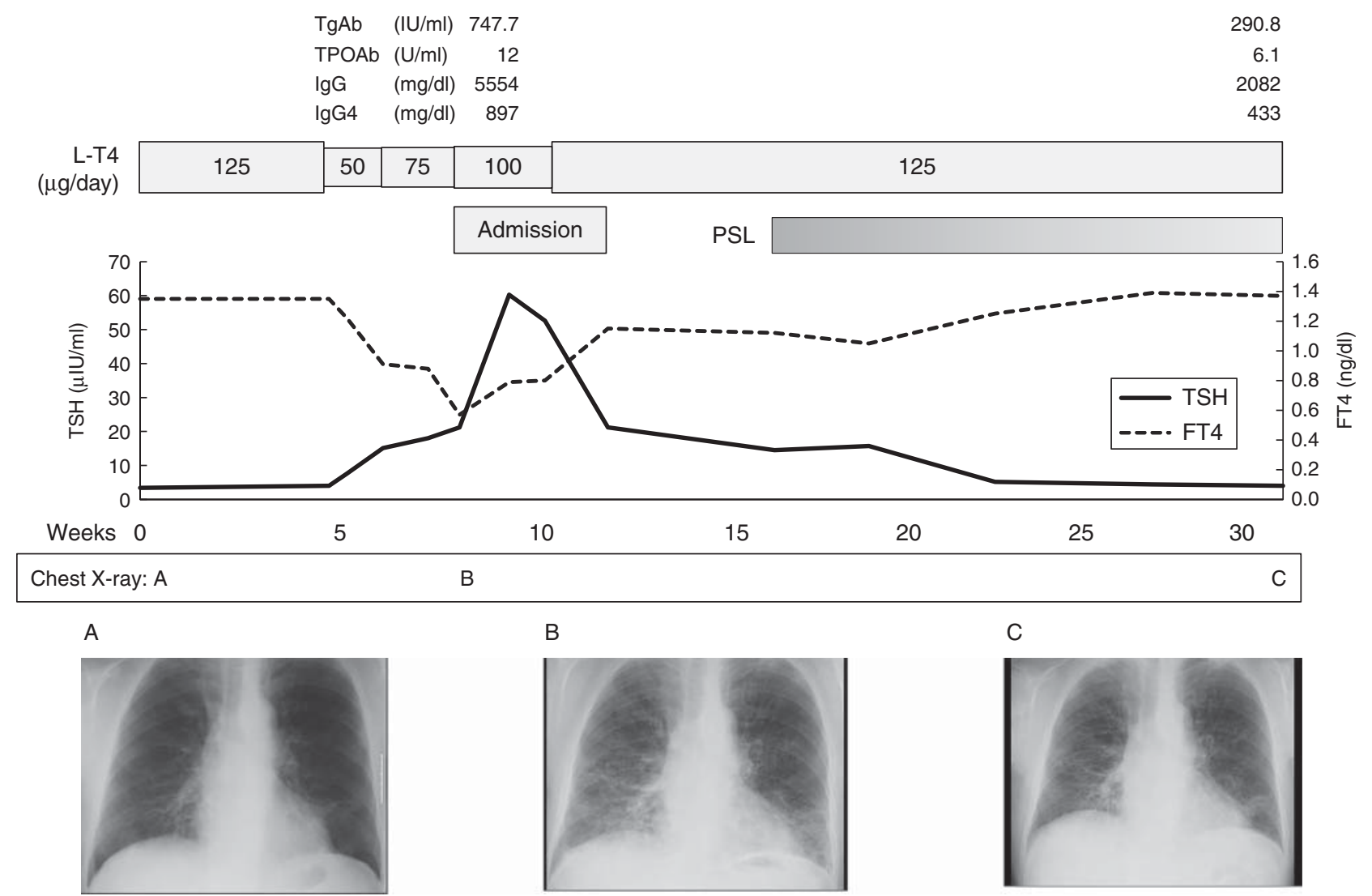

Figure 4

Clinical course and X-rays. No abnormal shadow was seen in chest X-ray 3 years before (A). On admission, consolidations in the both lower lobe was observed (B). In total, 10 weeks after the steroid therapy, the consolidations were improved (C).

\section{Treatment}

After commencement of oral PSL: $20 \mathrm{mg} /$ day, serum levels of complement bodies, such as C1q, CRP and sIL2R, were normalized by 6 weeks. On admission, L-T4 dosage was decreased to $50 \mu \mathrm{g} /$ day because of pseudo positivity in DLST. After re-evaluation of DLST and confirmation of normal response in DLST, L-T4 dosage was increased to
$125 \mu \mathrm{g} /$ day to obtain euthyroid, several weeks before PSL administration. However, TSH and FT4 levels were still subnormal. Notably, TSH and FT4 levels were normalized several weeks after PSL treatment (Fig. 4). Serum levels of $\mathrm{TgAb}$ and anti-thyroid peroxidase antibody (TPOAb) were paralleled with improvement of IgG and IgG4 levels and pulmonary lesion (Fig. 4C).
A

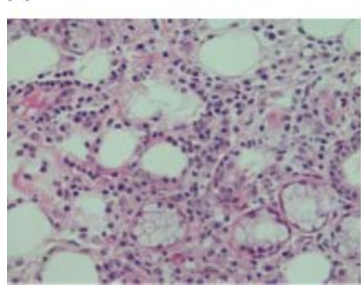

B

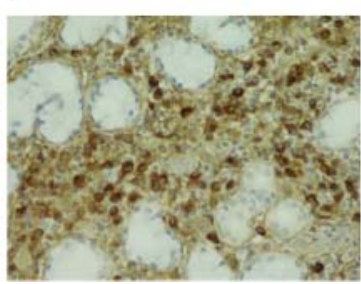

C

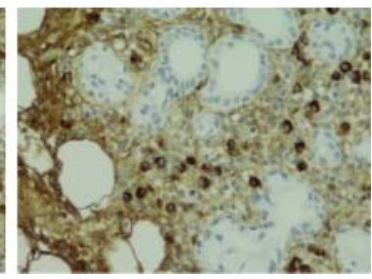

\section{Figure 5}

Histological examinations on the labial glands. In HE staining (A: $\times 400)$, lymphoplasmacytes and eosinophils infiltration with slight fibrosis were observed. Phlebitis was not clear. In IgG immunostaining (B: $\times 400)$, IgG positive plasmacytes were abundantly observed. In IgG4 immunostaining (C: $\times 400)$, IgG4 positive plasmacytes were also observed, and the ratio of positive staining of $\lg G 4 / \lg$ was $96 / 161=59.6 \%$. 


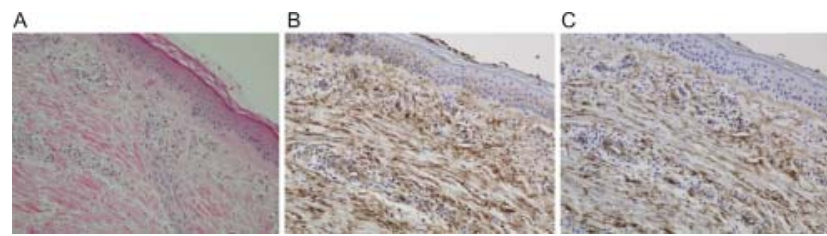

\section{Figure 6}

The result of skin biopsy of the purpura on the lower leg. In HE staining $(A: \times 200)$, in the shallow dermis, neutrophils, eosinophils and lymphocytes were observed around the small vessels. The vessel walls were mildly thickened. Extravasation of erythrocytes and infiltration of leukocytes in vessel wall and around small vessel were observed. Fibrin deposits and fibrinoid necrosis of small vessels were suspected. In IgG $(B: \times 200)$ and IgG4 (C: $\times 200)$ immunostaining, IgG or IgG4 positive plasmacytes were not seen, and IgG or IgG4 deposition on the vessel walls were not observed.

\section{Outcome and follow-up}

The dosage of PSL was decreased by $1 \mathrm{mg} / \mathrm{month}$, and after 1 year of follow-up period, no deterioration of IgG4-RD was seen.

\section{Discussion}

To the best of our knowledge, this is the first case of IgG4RD concomitant with leukocytoclastic vasculitis initially developed in the long course of HT. Based on the systemic manifestations in the lacrimal glands, salivary glands, pulmonary lesions, confirmed by histologically predominant IgG4 positive plasmacytes accompanied by serum IgG4 elevation, a diagnosis of IgG4-RD was made. IgG4related skin lesions can be categorized into seven subtypes (1), and the current case might be categorized as hypergammaglobulinemic purpura, bilateral asymmetrical palpable purpuric lesions on the lower extremities. Although increased IgG4 positive plasma cells were not confirmed in the skin lesion, the clinical course support the diagnosis with IgG4-related skin lesion. After the commencement of steroid therapy, improvements of the affected lesion paralleled with the decrease of serum IgG4 levels supported diagnosis with IgG4-RD. In addition, the amelioration of hypothyroidism after steroid therapy with fixed dosage of L-T4 supplementation suggested the presence of IgG4-RD-associated HT, which was a remarkable therapeutic outcome.

As known, HT shows Th1 predominance and antibody-dependent, cell-mediated cytotoxicity (3). However, in the current case, the development of IgG4-RD skewed to Th2 (IL4 and IL6) and Treg (TGF- $\beta$ ) polarization (Fig. 7), as reported previously in IgG4-RD (4).

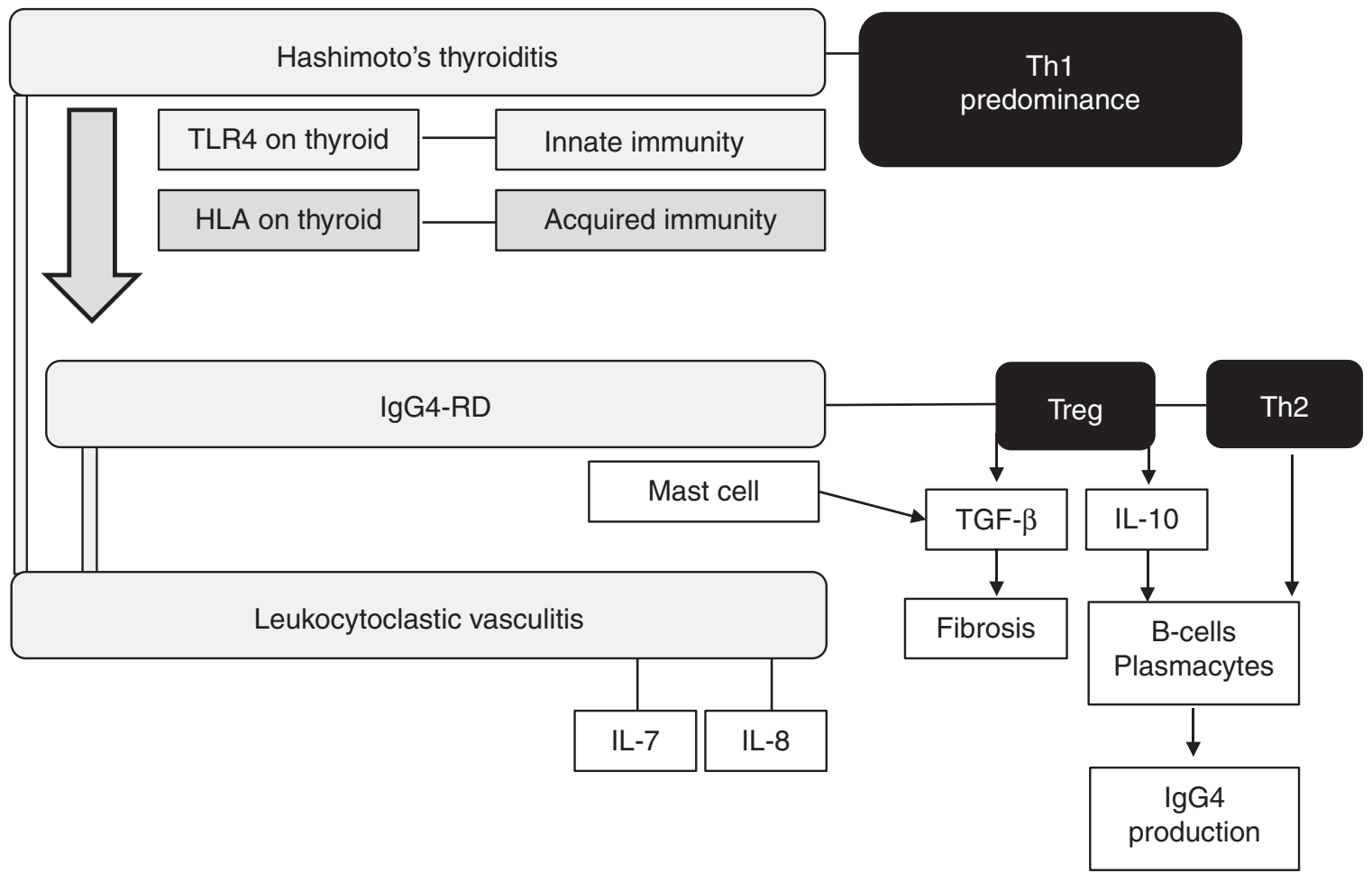

Figure 7

Immunopathological scheme of the relation of IgG4-RD, leukocytoclastic vasculitis and HT. Importantly, Th1-skewed balance was seen in PBMC, whereas serum levels of Th2 and Treg cytokines were elevated as described. 
IL7 plays a critical role in activation of memory Treg in skin (5). IL8, associated with skin lesion, accompanied by leukocytoclastosis (6). Thus, leukocytoclastic vasculitis seen in the current case might be related to IL7 and IL8 elevation. CCL2 promotes the polarization of Th2 and is upregulated in autoimmune thyroid disease (AITD) (7), as was seen in the current case. Interestingly, the ratio of Th1/Th2 cells in PBMC was increased (Table 1), similar to the cases of IgG4-RD (4) and severe HT (3). Given that serum Th2 cytokines and chemokine were increased, probably Th2 cytokines were produced not mainly from circulating $\mathrm{CD} 4{ }^{+} \mathrm{T}$-cell. Those curious cytokine imbalances may fluctuate in the time course (4). The proportion of Tregs was not increased in PBMC. Thus, the elevation of TGF- $\beta$ (Table 1) might be related to TGF- $\beta$ secretion from mast cells in the affected tissues (8). Taken together, combination of cytokines and chemokine measurements might predict the development of IgG4-RD and vasculitis in HT.

In the occurrence of HT in association with IgG4-RD concurrent with vasculitis raises the question of common etiologies, or antigens are involved in the pathophysiologic process. Notably, thyroid peroxidase (TPO) and myeloperoxidase (MPO) contain cross-reactive epitopes; thus, patients with HT and systemic vasculitis can possess autoreactivity to both TPO and MPO (9). With presence of broader areas of low echogenicity in thyroid, $\mathrm{TgAb}$ elevation, hypothyroidism and fine response to steroid therapy, his thyroid gland might be affected with IgG4-RD (2). The decrease of thyroid autoantibodies (TgAb and $\mathrm{TPOAb})$ titers during steroid therapy also supported the presence of IgG4-RD-associated HT (Fig. 4). Although co-existence of IgG4-RD-associated HT and non-IgG4RD-associated HT is suspected based on the long clinical course, initial treatment with steroid may improve thyroid autoimmunity as well as thyroid dysfunction.

The etiology in IgG4-RD has been unclear. Interestingly, toll-like receptor 4 (TLR4) is expressed on thyroid cells (10), and various antigens sensitization including gram-negative bacteria as ligand of TLR4 was proposed to be a trigger in the development of IgG4-RD (1). In addition, antigen exposures in HT are involved with cross-presentation on the surface of MHC molecules, and subsequently, epitope spreading of autoantigens and alloantigens occurs. It is well known that prolonged exposure of antigen may lead to IgG4 fraction predominance in HT. This IgG4-skewed balance in HT with long course may be related to the trigger of IgG4-RD. Therefore, thyroid autoimmunity, especially innate immunity as well as acquired immunity, was seemed to be involved in the development of IgG4-RD. As steroid is first-line treatment for IgG4-RD, the earlier intervention for IgG4-RD-associated HT may prevent its exacerbation. As described, as diagnostic approaches, the measurement of Th1, Th2, Treg cytokines, IL7, IL8 and CCL2 may help to distinguish these three entities (HT, IgG4-RD and leukocytoclastic vasculitis); thus, therapeutic aspects, antagonists or inhibitory molecules of certain cytokines and chemokines would be beneficial.

In conclusion, i) the current case exhibited rare presentation of IgG4-RD and its skin manifestation closely observed in the long course of HT, ii) measuring serum cytokines and chemokines levels as well as FACS analysis were demonstrated to be important to comprehend the development, progression and maintenance of IgG4-RD (Fig. 7), iii) this report also highlighted effective diagnostic approaches and treatment outcome in IgG4-RD-associated HT and iv) clinicians should pay attention to the development of IgG4-RD and vasculitis during long course of HT. Further investigations with increased number of cases during longer periods were warranted to establish diagnostic and therapeutic approaches to those entities.

\section{Declaration of interest}

The authors declare that there is no conflict of interest that could be perceived as prejudicing the impartiality of the research reported.

\section{Funding}

This article did not receive any specific grant from any funding agency in the public, commercial or not-for-profit sector.

\section{Author contribution statement}

All authors were involved in the preparation and writing of the manuscript.

\section{References}

1 Tokura Y, Yagi H, Yanaguchi H, Majima Y, Kasuya A, Ito T, Maekawa M \& Hashizume H 2014 IgG4-related skin disease. British Journal of Dermatology 171 959-967. (doi:10.1111/bjd.13296)

2 Takeshima K, Ariyasu H, Inaba H, Inagaki Y, Yamaoka H, Furukawa Y, Doi A, Furuta H, Nishi M \& Akamizu T 2015 Distribution of serum immunoglobulin G4 levels in Hashimoto's thyroiditis and clinical features of Hashimoto's thyroiditis with elevated serum immunoglobulin G4 levels. Endocrine Journal 62 711-717. (doi:10.1507/endocrj.EJ15-0157)

3 Nanba T, Watanabe M, Inoue N \& Iwatani Y 2009 Increases of the Th1/Th2 cell ratio in severe Hashimoto's disease and in the proportion of Th17 cells in intractable Graves' disease. Thyroid 19 495-501. (doi:10. 1089/thy.2008.0423)

4 Okazaki K, Yanagawa M, Mitsuyama T \& Uchida K 2014 Recent advances in the concept and pathogenesis of IgG4-related disease in the hepato-bilio-pancreatic system. Gut and Liver 8 462-470. (doi:10.5009/ gnl14107) 
Endocrinology,

Diabetes \& Metabolism

CASE REPORT
H Minamino and others

Association of IgG4-RD and

vasculitis with $\mathrm{HT}$
ID: 16-0004; March 2016

DOI: 10.1530/EDM-16-0004
5 Gratz IK, Truong HA, Yang SH, Maurano MM, Lee K, Abbas AK \& Rosenblum MD 2013 Cutting Edge: memory regulatory t cells require IL-7 and not IL-2 for their maintenance in peripheral tissues. Journal of Immunology 190 4483-4487. (doi:10.4049/jimmunol.1300212)

6 Grunwald MH, Shriker O, Halevy S, Alkan M \& Levy R 1997 Impaired neutrophil functions in patients with leukocytoclastic vasculitis. International Journal of Dermatology 36 509-513. (doi:10.1046/j.13654362.1997.00195.x)

7 Heul-Nieuwenhuijsen L, Padmos R, Drexhage R, de Wit H, Berghout A \& Drexhage HA 2010 An inflammatory gene-expression fingerprint in monocytes of autoimmune thyroid disease patients. Journal of Clinical Endocrinology and Metabolism 95 1962-1971. (doi:10.1210/ jc.2009-1455)
8 Takeuchi M, Sato Y, Ohno K, Tanaka S, Takata K, Gion Y, Orita Y, Ito T, Tachibana T \& Yoshino T 2014 T helper 2 and regulatory T-cell cytokine production by mast cells: a key factor in the pathogenesis of IgG4related disease. Modern Pathology 27 1126-1136. (doi:10.1038/modpathol.2013.236)

9 Haapala AM, Hyöty H, Parkkonen P, Mustonen J \& Soppi E 1997 Antibody reactivity against thyroid peroxidase and myeloperoxidase in autoimmune thyroiditis and systemic vasculitis. Scandinavian Journal of Immunology 46 78. (doi:10.1046/j.1365-3083.1997.d01-90.x)

10 Nicola J, Vélez M, Lucero A, Fozzatti L, Pellizas CG \& Masini-Repiso AM 2009 Functional toll-like receptor 4 conferring lipopolysaccharide responsiveness is expressed in thyroid cells. Endocrinology $150500-508$. (doi:10.1210/en.2008-0345)

Received in final form 7 January 2016

Accepted 19 February 2016 